\title{
Risk Assessment Issues Associated with Cleaning up Inactive Hazardous Waste Sites
}

\author{
by Elizabeth L. Anderson, Ph.D., ${ }^{1}$ \\ Paul C. Chrostowski, Ph.D., \\ Judy L. Vreeland, M.S
}

\section{Introduction}

The broad practice of using risk assessment approaches for the evaluation of suspected human carcinogens is about 12 years old. The primary departure point was the announcement by the U.S. Environmental Protection Agency (EPA) which adopted guidelines for assessing the risk of carcinogens and a policy to regulate suspect carcinogens based essentially on a risk management approach. The scientific basis was derived from the earlier experience of assessing the risk of health impacts from radiation exposure. From a practical standpoint, the use of risk assessment for carcinogens has received broad and general endorsement. The early use of risk assessment of carcinogens relied heavily on replacing the uncertainties in the risk assessment process with very conservative assumptions to make sure that, in no case, would the risk be underestimated. As the practice of risk assessment has become widespread, considerable attention has been focused on improving the scientific basis for evaluating each step of the risk assessment process: the weight-of-evidence indicating likely carcinogenicity, the dose-response relationships, and the environmental exposures. Chemicals which are thought to cause health or ecological effects through threshold mechanisms are also being evaluated by risk assessment approaches. More attention has been focused on the scientific relationships that underlie the characterization of suspect carcinogens and their dose-response relationships including extrapolation from animals to humans and from high dose to low dose; far less attention has been focused on the exposure assessment which can impact the outcome of the quantitative risk assessment by certainly as much as the assumptions in the dose response extrapolation part of risk assessment. Recent advances in both areas when applied to hazardous waste sites risk assessment, can substantially alter the outcome of the site specific risk assessments. This paper will provide an overview of scientific developments in risk assessment and describe how the use of these improved scientific data may alter the outcome of the standard Superfund risk assessment approaches.

${ }^{1}$ E. L. Anderson, President, P. C. Chrostowski, Vice President, J. L. Vreeland, Senior Associate, ICF-Clement Associates, Inc., 9300 Lee Highway, Fairfax, Virginia 22031-1207. (703) 934-3500 


\section{Risk assessment}

\subsection{An Overview of the Process}

In 1976, EPA adopted the first policy for the use of risk assessment of toxic chemicals which were suspected of being human carcinogens and accompanied this policy statement with guidelines for the scientific risk assessment process (EPA 1976; Albert et al. 1977). These guidelines were adopted in response to the need of a major regulatory agency to develop a means for regulating the presence of hundreds of suspected carcinogens in the environment under numerous environmental legislative statutes which had been adopted by Congress. In short, it was obvious that EPA could not regulate all suspected carcinogens which were being identified in rapid succession as environmental contaminants to a zero risk level as had been the risk goal of the Food, Drug and Cosmetic Act's Delaney Clause. Such a goal had clearly been the objective of the strong environmental movement which characterized the first half of the decade of the 1970's. The adoption of guidelines for risk assessment, with the implication that EPA planned to accept residual risk as a regulatory policy, was initiated under the watchful eyes of the scientific community, the regulated community, and the environmental communities. In short, there was considerable skepticism about the approach as a basis for public policy because of the substantial scientific uncertainties, particularly in quantitative risk assessment. After the EPA action, several other endorsements followed. The Inter-Agency Regulatory Liaison Group (IRLG) adopted similar scientific principles in 1979 (IRLG 1979). These guidelines were followed by the report of the National Academy of Science which endorsed the use of risk assessment and provided descriptive terms for each step of the risk assessment process which have now been adopted as a common vocabulary (NAS 1983). In addition, the Office of Science and Technology Policy (OSTP) published similar scientific principles in 1985 (OSTP 1985) and EPA has updated its earlier guidelines (EPA 1986). In short, the application of risk assessment to toxic chemicals for the evaluation of scientific evidence that indicates that a chemical might be a human carcinogen and also provides information as to the magnitude of current and anticipated public health impacts has been endorsed and described in many forums and also has been the subject of discussion in many scientific conferences.

The process is generally described in four steps: hazard identification, dose-response modeling, exposure assessment, and overall risk characterization (NAS 1983). In practice, over the last 12 years, the hazard identification step in risk assessment has relied on all of the available human, animal, and/or in vivo or in vitro data to describe the weight-of-evidence that indicates that a chemical might be a human carcinogen. At various times, the weight-of-evidence has been stratified according to either the International Agency for Research on Cancer (IARC) criteria (IARC 1982) or the more recent EPA stratification scheme (EPA 1986a) for assigning a category to the weight-of-evidence. While these two categorical schemes are very closely related, the EPA scheme expands on the inadequate evidence labeled Category 3 in the IARC criteria to include three additional categories: $C$ to indicate evidence that constitutes the category of "probable" carcinogen for humans; D to indicate inadequate testing; and $\mathrm{E}$ to indicate negative evidence.

Dose-response modeling has largely followed a linear non-threshold hypothesis for lowdose extrapolation as a basis for defining a "plausible upper limit" on the risk, meaning that the risks are unlikely to be higher but could be considerably lower (Crump et al. 1977; Crump and Watson 1979; Crump 1981; OSTP 1985; EPA 1986a). This model relies on the 
possibility that any suspected carcinogen can induce cancer by a single hit phenomenon and makes no distinction for different biologically based mechanisms of cancer induction. To date, other models which haved variously been suggested for low-dose extrapolation from high dose data have been empirically based models which seek statistically to define the best shape to the dose-response curve; they have not been based on data which seek to describe the biological events which lead to cancer.

Other assumptions, such as those used to extrapolate animal responses to humans, have been adopted which also have been chosen to be protective where scientific information was lacking (e.g., surface area is often chosen as the conversion factor rather than body weight). Dose is assumed to be synonymous with exposure, unless there are data to the contrary. Other conservative assumptions have also been chosen including, for example, the interpretation of the significance of benign tumors, which can lead to malignancy.

Exposure assessment likewise has followed a conservative trend. Generally, "maximum plausible levels" of chemical exposure have been used in risk assessments, sometimes in conjunction with "average exposure" estimates. An example of a frequently used conservative assumption is that an individual is exposed for a lifetime of 70 years unless there is evidence to the contrary. In practice, the overall risk characterization has relied on a ranking of the weight-of-evidence which placed considerable weight on any tumor response in animals and sought to quantitatively describe the risk to current or anticipated exposed populations as an "upper-bound risk" based on "maximum plausible" exposure estimates. The first decade of experience with carcinogen risk assessment has been studied both from the scientific standpoint and the use of risk outcomes in public policy decisions (Anderson and CAG 1983). In short, if scientists have been successful in the past describing risk assessment as "upper bound" estimates reflecting "maximum plausible exposures," then as better science is developed to fill the gaps of uncertainty, risk assessments should be expected to become less conservative.

\subsection{Current trends}

Historically, protective assumptions replaced uncertainties; in some cases, uncertainty is now being replaced by improved scientific information. In the area of weight-of-evidence, fresh consideration is being given to the weighting of evidence at high dose and its appropriateness for low-dose weighting. For example, in the Carcinogen Assessment Group's risk assessment of ethylenethiourea (ETU) (EPA/CAG 1977), the uniqueness of the observation of rat thyroid tumors was discussed in the context of having a threshold, namely that these tumors resulted from suppression of thyroid activity only after the administration of a sufficiently high dose. Currently, the rat response is being examined to determine whether environmental exposure levels are likely to approach those that could be expected to elicit the rat thyroid tumor response; if not, it may be appropriate only to factor the mouse liver tumor response results into the weight-of-evidence determination for environmental exposure levels. Other chemicals are similarly being reviewed for their relevance to human exposure because of mechanism of action, tumor type observed, dosing levels used, or metabolic and pharmacokinetic differences between humans and laboratory test animals.

The improvements in dose-response modeling probably represent the most dramatic departure from practices of the last 12 years. There is a clear effort by regulatory agencies to seek a biological basis for the development of more accurate estimates of risks expected 
to occur at environmental exposure levels. This effort represents a substantially different approach from applying empirical formulas to estimate low-dose responses from high-dose data; rather the attention is focused on the importance of research data that may guide lowdose modeling efforts. Such an approach provides, at a minimum, an indication of the extent to which the "plausible upper bounds" may be overestimating risk for particular chemicals. Early efforts to define more accurate estimates of risk began at EPA in early 1985 and have culminated in the development of a generic approach using a two-stage model. This model adapts the clinical observations of Moolgavkar and Knudson (1981) to parameters involving exposure to toxic chemicals. The effort was first undertaken by EPA's Risk Assessment Forum and was ultimately published in the Journal of Risk Analysis in early 1987 (Thorslund et al. 1987). Thus far, EPA has proposed two important decisions in line with the trend toward less conservatism in dose-response modeling. Both of these decisions were discussed in a recent New York Times article (Shabecoff 1988). For example, the EPA's Risk Assessment Forum has recommended lowering the arsenic ingestion potency by approximately an order of magnitude (Levine et al. 1987; Moore 1987) based on modifications in dose-response calculation methodology and better estimates of the exposure involved in the epidemiology studies that were the basis for the evaluation. There is a further consideration of reducing the potency of arsenic by ingestion by still another order of magnitude to reflect the fact that skin cancer caused by arsenic ingestion is less likely to lead to death than is lung cancer induced by inhalation. Considerations of the latter raise the issue as to whether or not treatability, survival, and severity should be routinely considered as a part of the risk assessment process, and in particular the potency evaluation. In addition, EPA has proposed to downgrade the potency of dioxin based on several factors but most importantly, the use of the two-stage model of carcinogenesis for modeling the promoting activity of dioxin which indicates that the potency of dioxin may be two orders of magnitude or more less than the potency defined by the linear nonthreshold model for low doses (T. W. Thorslund and G. Charnley, in preparation). This work was prompted by recommendations of the EPA Science Advisory Board and is still under consideration (EPA/OPTS 1986b).

The two-stage model of carcinogenesis has also been applied to several other chemicals with similar outcomes. For example, the model has also been applied to chlordane and heptachlor, and methylene chloride (T. W. Thorslund et al. 1988, private communication). While the mechanisms in each case differ, the outcomes of the model are to indicate most often several orders of magnitude lower potency at low dose than predicted by the linear nonthreshold model at the "plausible upper bounds."

Additional applications of the biological model have involved the polycyclic organic compounds. Past practices have used the potency of benzo(a)pyrene as a unit equivalency to all other potentially carcinogenic polycyclic organic compounds, greatly overestimating risk. This practice has continued in spite of the fact that comparative potency methods have been developed for other chemical classes, such as the dioxins. When assembled in the aggregate, several laboratory studies provide a more substantial basis for developing a comparative potency approach for PAHs (M. M. L. Chu and C. W. Chen 1984, unpublished; Thorslund et al. 1986). In addition, the shape of the dose response curve for benzo(a)pyrene itself has been reevaluated. Benzo(a)pyrene is a genotoxic agent as indicated by a linear rate of DNA adduct formation that parallels exposure. The tumor dose response data do not parallel DNA adduct formation, however, but appear to fit a quadratic equation, indicating 
that two events are probably necessary to induce the response. EPA's initial cancer potency estimate for benzo(a)pyrene does not reflect this relationship. The comparative potency approach for other polycyclic compounds, together with a revised dose-response curve for benzo(a)pyrene, has been used to accurately predict tumor outcomes in bioassays of chemical mixtures, which is not possible using upper-bound estimates (Thorslund et al. 1986). Another example of a chemical which may require two events to produce a cancer outcome is benzene. Current investigations are examining the mechanistic data, which indicate benzene causes chromosome damage which is thought to be responsible for the chromosomal deletions and rearrangements observed in leukemia patients. This relationship implies that, although linearity may establish a plausible upper bound on human leukemia risk from benzene exposure, a quadratic relationship may be more appropriate to estimate the actual risk. Should this turn out to be the case, the risk from low dose exposure to benzene would be considerably lower than previously estimated (T. W. Thorslund and G. Charnley 1988, private communication).

A great deal of attention is also being focused on the metabolic and pharmacokinetic data to estimate actual levels of chemical exposure to the target tissue. In extrapolating animal data to humans, the effective dose in the animal studies has always been assumed to be the dose that the animal was exposed to by route-administered-dose. As our ability to describe the actual dose to the target tissue in the animal improves, so will our ability to extrapolate animal responses to humans. In addition, the importance of pharmacokinetic data to define the significance of human exposure in the environment is exceedingly important.

Less progress has been made for threshold pollutants. While attention is currently focused on developing biologically based dose-response curves to better describe the threshold dose for disease causation, by the majority of these chemically induced effects are still described by applying safety factors to no-observed-effect levels (NOELs) from animals studies or for some few chemicals, describing the effective dose for observations in humans, e.g., lead. In either case, the results are uncertain and the outcomes subject to scientific debate.

Of equal importance, trends in exposure assessment research are also leading to improved estimates of population exposures which provide a better foundation for current and projected exposures. Traditional practices have relied heavily on generic models to describe exposure to human populations. EPA has developed generalized dispersion models for describing air transport and similar generalized dispersion models for surface and groundwater. The overall impact of these dispersion models has been to provide conservative estimates of exposure.

The use of generalized models provides a practical approach for widespread exposure estimation by regulatory agencies because it would be highly impractical for a national agency to evaluate site-specific parameters for every source. For important cases, however, it is possible to estimate actual parameters that may refine the estimates obtained by generic modeling. An example is the risk assessment of the ASARCO smelter in Tacoma, Washington which was conducted by EPA (Patrick and Peters 1985). The use of generalized dispersion modeling using the human exposure model (HEM) (which assumes a flat terrain, an immobile population, and uses meteorological data from the closest weather station), when coupled with the dose-response curve, estimated a maximum individual risk of about $1 \times 10^{-1}$ for populations living near the smelter. Subsequently, a local study was conducted 
which permitted the use of several site-specific assumptions including a more accurate description of the actual terrain, local meteorological data, and better emissions information. The outcome was to lower the exposure assessment and the overall risk about an order of magnitude. This brought the risk into closer alignment with the limited monitoring data which was available for the ambient air.

The same phenomenon has been observed when comparing estimates using generalized dispersion models for groundwater with estimates which rely on site specific parameters. For example, in Figure 1, the generalized dispersion model, the vertical horizontal spread (VHS) model using EPA default values overestimates the risk by a factor of 5.7 when compared to the results from the more complex equation which incorporates measured site values (Domenico and Palciauskas 1982; EPA 1985). Another important area which has sharpened exposure estimates and practically has lowered the outcome from exposure assessment by several orders of magnitude and, thus the quantitative risk assessment, has been considerations of bioavailability. For example, dioxin was originally assumed to be $100 \%$ biologically available in soil. Recent studies, however, have demonstrated that dioxin is only partially available, $>0.5 \%-85 \%$ depending on soil type (Umbreit et al. 1986). In practice, it has been our experience that dioxin is mostly available in the range of $15-50 \%$ (P.Chrostowski 1988, private communication). Dioxin in fly ash also was originally assumed to be up to $100 \%$ available. Recent studies have found that this is not correct but rather that dioxin in fly ash is biologically available between $0.1 \%$ and $0.001 \%$ (van den Berg et al. 1986). The bioavailability issue is now being commonly investigated in many different situations where the availability in soil and fly ash is important to the outcome of the risk assessment.

Although improving the scientific information available for site-specific exposure assessment tends to lower the overall outcome of the exposure assessment and thus the risk assessment, there are important exceptions. For example, a recent paper which addressed the issue of risk associated with inhaling volatile organic chemicals from contaminated drinking water during shower activity (Foster and Chrostowski 1987) indicated that as much as half or more of the total body risk could be associated with the shower exposure rather than with the drinking water exposure. In addition, recent improved methods for modeling the actual deposition of particulate matter from stationary sources tends to raise the risk compared the earlier EPA air transport models which assumed that both large and small particles bounced from the surface of the earth in very similar ways and were carried from the site by air transport. The more recent models take into account that the small particles deposit on the surface and are not so readily transported (Sehmel and Hodgson 1979). Also, closer attention to chemical conversions may tend to raise or lower the risk; for example, trichloroethylene is converted under anaerobic conditions to vinyl chloride which has a higher potency value by ingestion than does trichloroethylene (Parsons et al. 1984; Cline and Viste 1984). Recognition of this conversion raises the overall risk assessment for circumstances which appropriately are evaluated by these methods.

Numerous other refinements in exposure assessment are also being incorporated in the risk assessment process, for example, use of human biological data to assist in exposure estimation, better descriptions of life style for subpopulation groups, the use of statistical methods to describe likely exposure below detectable limits, and the use of pharmacokinetic data to describe the actual dose to target tissue. These developments rely on advancing research in multiple disciplines for use in the practical consideration of human exposure. 


\section{Applications to hazardous waste site risk assessment}

Wastes site risk assessment practices have roughly paralleled the conservative (public health protective) approaches of risk assessment approaches over the last dozen years. The majority of this experience has been gained from the investigation of Superfund sites according to the standard EPA Superfund Manual and related guidelines (USEPA 1985a, $1986 \mathrm{c}, \mathrm{d})$. In these investigations a risk assessment is a formalized methodology applied to determine the potential for human health and environmental impacts associated with a site under the no action alternative or to evaluate the potential benefits from remedial alternatives.

Generally, the initial step in conducting a risk assessment involves a review of all available site environmental monitoring data in order to select potential chemicals of concern on which the assessment will focus. At this step, chemical measurements with inadequate quality assurance/quality control or chemicals that are present as part of natural background may be rejected for inclusion in the assessment. The next step, hazard identification, involves identifying chemical-specific human health and ecological effects criteria. This may involve an evaluation of available data, including epidemiology, animal bioassay studies, and in vivo and in vitro studies. In the absence of human data to describe low-dose effects, the frequently used approaches for dose-response characterization are for "threshold" (non-carcinogenic) and "non-threshold" (carcinogenic) effects. These approaches generate numerical health effects criteria to be used in the calculation of risk. While some guidance levels generally exist for most toxic chemicals, further scientific work may be warranted. Recent reconsideration by the USEPA of potency factors for arsenic, dioxin, and polycyclic aromatic hydrocarbons are good examples. Following hazard identification, potential pathways by which human populations may be exposed under current or potential future land-use conditions are identified. An exposure pathway is composed of the following four elements: 1) a source and mechanism of chemical release to the environment; 2) an environmental transport medium (e.g., groundwater) for the released chemical, and/or a mechanism of transfer of the chemical from one medium to another; 3) a point of potential contact of humans or biota with the contaminated medium (the exposure point) and; 4) an exposure route (e.g., ingestion) at the exposure point. All four of these elements must be present for a pathway to be considered complete. To evaluate exposure at an exposure point the concentration of chemicals of concern must be evaluated. Many times these are actual measured concentrations, however when they have not been measured, or to estimate future concentrations expected to occur over a longer time (i.e., a 70 year lifetime), or at exposure points not previously investigated, environmental fate and transport modeling may be necessary. For Superfund sites, once concentrations of chemicals of concern at the exposure points have been determined, they are compared with "applicable or relevant and appropriate requirements" (ARARs). When ARARs are not available for all chemicals in all media, quantitative risk estimates are developed by combining the estimated intakes of potentially exposed populations (often derived using conservative assumptions regarding chemical concentrations, exposure duration, exposure frequency, and the efficiency of absorption in biological media of chemicals) with either existing health effects criteria or improved evaluations based on more recently available data and methods. Conservative assumptions are generally made in risk assessments to compensate for uncertainty and to explore the potential for adverse health effects using conditions that tend to overestimate risk so that the final estimates will usually be near or higher than the upper end of the range 
of actual exposures and risks. Greater uncertainty in the site-specific data base generally leads to more extensive reliance on conservative assumptions; conservative (i.e., protective) assumptions are chosen to make certain that risks will not be underestimated. Because there is uncertainty, risk assessments generally do not present an absolute estimate of risk; rather most risk assessments establish plausible upper bounds on risk to indicate the potential for adverse impacts. Thus, risk assessments are more useful where data are available to narrow uncertainties and permit the most accurate descriptions of risk possible. In the absence of such data, conservative approaches which provide upper bound risk estimates present clear guidance for the evaluation of low risk (i.e., that even at the upper bounds the risks are low and therefore most often do not warrant regulatory attention) but are less instructive for remedial prescription where the social and economic costs are high.

\subsection{Potential pathways of exposure to contaminants}

All pathways of exposure are considered: groundwater, surface water, soil, and air. The pathway that is most often of greatest concern is groundwater. For purposes of discussion, this paper focusses on this route as an example of an exposure route evaluation.

To evaluate exposure to groundwater, standard intake assumptions are generally employed. These are that an average adult ingests 2 liters of water a day over a 70 year lifetime and that the average body weight over the exposure period is 70 kilograms, unless there are clear data to define alternative choices. For example, these assumptions can be arguably too stringent or in some cases, such as outdoor workers in an arid climate, they may not be stringent enough. If the demographics and activity patterns of the population are known, more accurate intake assumptions may be used which will often diminish risks.

Additionally, inhalation exposures to contaminants in groundwater may occur through use of water in day to day activities such as cooking, bathing, washing of dishes and clothes, or showering. Dermal exposures are also possible. Although many of these exposures may be dependent upon individual water-use patterns, exposure through showering may be quantified using the model of Foster and Chrostowski (1987). For many volatile organics quantification of the additional risks through inhalation of contaminants while showering may be similar to the risks associated with ingestion. In some instances, risks from all inhalation activities combined may be greater than those associated with ingestion, especially if the chemical involved is more toxic by the inhalation route (e.g., 1,1-dichloroethylene). Dermal exposures are generally small compared to ingestion or inhalation, although they may be substantial when chemicals which are absorbed with a high efficiency (e.g., dimethyl sulfoxide) are involved. Failure to assess these pathways could lead to groundwater risk management decisions not protective of public health or associated with an inaccurate representation of liability.

In many locations groundwater discharges into surface water bodies create additional potential pathways of exposure. This is a particular concern for water bodies that are of moderate size (i.e. that have sufficient flow to support aquatic life and are not so large in volume as to dilute concentrations of contaminants discharging in groundwater to insignificant concentrations). Contaminants that have high octanol-water partition coefficients $\left(\mathrm{K}_{\mathrm{ow}} \mathrm{s}\right)$ have a potential to bioaccumulate and generally are of particular concern. These chemicals may not only be toxic to aquatic life but may potentially cause risks to other organisms higher in the food chain or to humans that ingest fish from these surface water 
bodies on a regular basis. Additionally surface water bodies may be used for other recreational activities such as swimming, or for drinking water supplies; these may create additional exposure pathways to contaminants in groundwater.

An added consideration is needed to provide an assessment of anticipated exposures. For example, a change in local pumping conditions due to the installation of a new well (particularly one that has a high yield such as an industrial or municipal well) may have an influence on contaminant migration or groundwater which is not currently a drinking water source may become a source in the future.

In evaluating potential exposure to contaminants present in groundwater, it is important to define the aquifer(s) to be evaluated. This may be a particular concern when a source area is underlain by more than one aquifer that may potentially be used for a water supply. The potential exposure associated with the use of each one of the aquifers may be evaluated individually. However in many instances, particularly in areas with fractured bedrock, domestic wells are open to more than one water bearing zone and estimation of the potential future concentrations in these wells would be dependent upon the well construction. This type of information in some instances is not readily available, and is difficult to estimate for hypothetical future wells.

USEPA's groundwater strategy (USEPA 1986e) suggests that risks associated with potential water supplies should be evaluated as if the water supplies were actually in use. Thus aquifers with natural mineral contents low enough for potability and with high enough potential yields are all considered potable whether in current use or not.

\subsection{Approaches to quantifying exposure and risks associated with contaminants in groundwater}

Site specific information is essential in evaluating the potential risks associated with exposure to contaminants present in groundwater. For a screening approach, general hydrogeological parameters can often be obtained from available publications (e.g., Freeze and Cherry 1979, Walton 1985). Use of these general default parameters, however, could lead to an inaccurate portrayal of risk. However, to refine groundwater models with concomitant refinement of risk estimates, site specific information with regard to the hydrogeological characteristics and potential source areas must be well defined. With regard to hazardous waste sites, there is frequently little to no information available regarding the history of chemical disposal in potential source areas, thus conservative assumptions are generally made with regard to the nature, extent, frequency, and duration of chemical release to the subsurface.

Oftentimes estimation of concentrations of chemicals of concern in the groundwater begins with evaluating the transport of contaminants from the source, which is often soil, through the unsaturated zone. This can be done through a variety of approaches ranging from a simplified steady-state soil-water partitioning model to time dependent models that consider linear adsorption/desorption without accounting for dispersion (Enfield et al 1982) or more complex compartmental numerical models that incorporate time-varying transport, advection and dispersion such as the Pesticide Root Zone Model (USEPA 1984). Applying these models to the same site may result in soil pore water concentrations that may vary by as much as several orders of magnitude. 
The output from the unsaturated zone models may then be coupled with groundwater models to predict concentrations of chemicals of potential concern at potential exposure points. The groundwater models may range from simplified mass-balance mixing models through analytical solutions of transport equations to complex three dimensional numerical models. In some instances simplified models used for screening purposes show that even using conservative assumptions the estimated concentration at a potential exposure point may not be associated with a risk and the conservative assessment may be sufficient. However refinement of conservative assumptions is often necessary to ensure that the evaluation is realistic and that remedial actions will not be undertaken needlessly.

An example of the importance of applying site specific parameters to a hazardous waste site in the context of groundwater solute transport modeling can be instructive. For example, the Vertical Horizontal Spread (VHS) Model (USEPA 1985a, 1986f) is a steady state groundwater model in which the only attenuation mechanism is vertical and horizontal spreading; it neglects longitudinal dispersion, and chemical degradation kinetics. Application of the VHS model to a particular site using USEPA fixed default values resulted in a ratio of concentration at the exposure point to source concentration $\left(C / \mathrm{C}_{\mathrm{O}}\right)$ of 0.34 . However, a refinement of the model incorporating vertical and horizontal dispersion coefficients, and site specific parameters resulted in a $C / \mathrm{C}_{0}$ ratio of 0.06 . Thus the generic USEPA model overestimated the concentrations and risks at the exposure point by actor of 5.7 (Figure 1).

Figure 1

Ground Water Modeling

1. VHS model using simple equation, EPA fixed default values

$$
\begin{aligned}
\frac{\mathrm{C}}{\mathrm{C}_{\mathrm{o}}} & =\operatorname{erf}\left[\frac{\mathrm{Z}}{2(\mathrm{DY})^{0.5}}\right] \operatorname{erf}\left[\frac{\mathrm{X}}{4(\mathrm{DY})^{0.5}}\right] \\
& =0.34
\end{aligned}
$$

2. VHS model using complex equation, measured site values

$$
\begin{aligned}
\frac{\mathrm{C}}{\mathrm{C}_{\mathrm{o}}}=\frac{1}{4} & {\left[\operatorname{erf}\left(\frac{\mathrm{z}+\mathrm{Z}}{2\left(\mathrm{D}_{\mathrm{Z}} \mathrm{Y}\right)^{0.5}}\right)-\operatorname{erf}\left(\frac{\mathrm{z}-\mathrm{Z}}{2\left(\mathrm{D}_{\mathrm{Z}} \mathrm{Y}\right)^{0.5}}\right)\right] } \\
& {\left[\operatorname{erf}\left(\frac{\mathrm{x}+\mathrm{X} / 2}{2\left(\mathrm{D}_{\mathrm{x}} \mathrm{Y}^{05 .}\right.}\right)-\operatorname{erf}\left(\frac{\mathrm{x}-\mathrm{X} / 2}{2\left(\mathrm{D}_{\mathrm{x}} \mathrm{Y}\right)^{0.5}}\right)\right] } \\
= & 0.06
\end{aligned}
$$

Conclusion: EPA method overestimates concentration at exposure point by factor of 5.7 . 
In many instances the steady-state assumption is not applicable. At another site, site specific groundwater modeling incorporating chemical decay and source decay illustrated that assuming that the observed concentration persists over 70 years could result in a substantial overestimation of the risk. For example, work completed by our scientist at a site in California indicated an "upper-bound" lifetime risk associated with ingestion of water containing trichloroethylene (TCE) in an aquifer to be as high as a $10^{-3}$ risk (Figure 2).

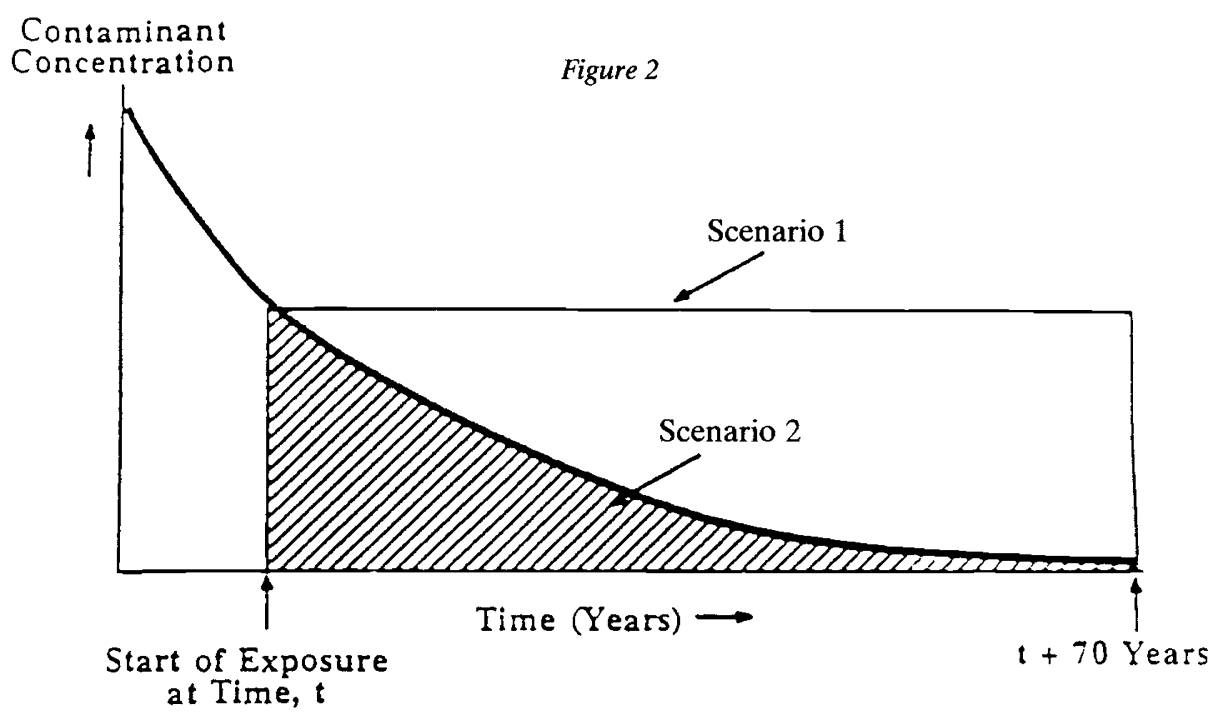

Scenario $I$ assumes decay in contaminant levels until start of consumption and then a lifetime exposure to a constant concentration of the contaminant.

Scenario 2 assumes decay in contaminant levels both before and during the period of exposure.

This level is associated with a 70-year lifetime exposure via exposure to drinking water from the contaminated aquifer. Scenario 2 in Figure 2 describes the decline in risk associated when hydrogeology models are applied to the site; the model assumes that the source of contamination has been removed. In Figures 3 and 4 the monitoring well data are displayed and likewise the risk comparison over time given the ability to model the area. In this particular circumstance, remedial action was being considered which would cost in the million dollar range and require a number of months to install. If the hydrogeology models are correct, the theoretical risk could be lowered considerably over the first 18 -month period given the natural ability of the hydrogeology of the area to remove the contamination. Caution, however, should be exercised in assuming that the source has been removed because recent publications indicate that in some circumstances some chemicals may remain entrapped in soil micropores and thereby provide a slow, diffuse release (Sawhney et al. 1988).

As the concentration of a particular compound present in groundwater may decrease through natural processes such as biodegradation, the total risk resulting from exposure to the groundwater may not necessarily decrease. Some compounds are transformed into 
Figure 3

Groundwater Monitoring data

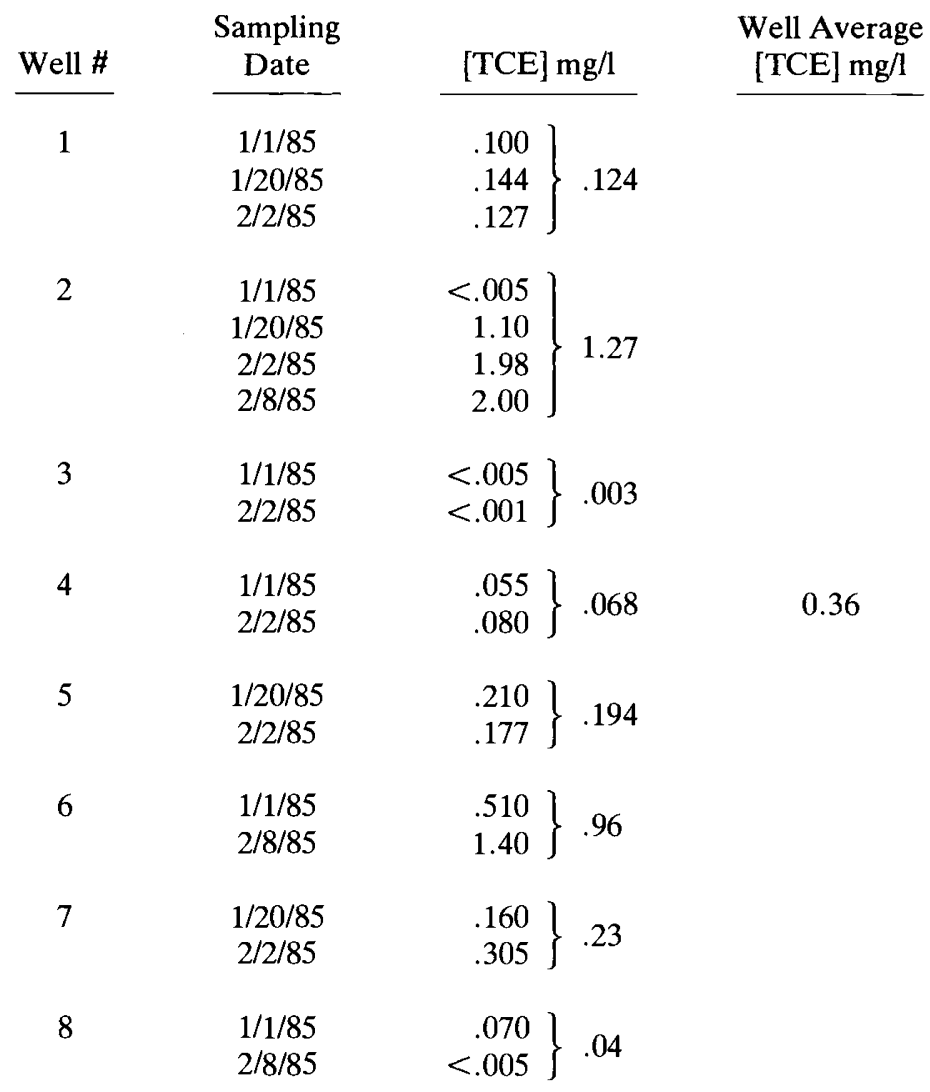

more toxic compounds through biotransformation. For example, under anaerobic conditions, some halogenated aliphatics have been found to undergo reductive dechlorination (Bouwer et al. 1981, Kobayashi and Rittmann 1982, Vogel and McCarty 1985). The transformation is sequential, with, for example tetrachloroethylene yielding first trichloroethylene (TCE) and, ultimately vinyl chloride (Parsons et al. 1984, Cline and Viste 1984). Trichloroethylene is categorized as a probable human carcinogen with a cancer potency factor of $0.011 \mathrm{mg} / \mathrm{kg} /$ day whereas vinyl chloride is a known human carcinogen with a cancer potency factor that is approximately two orders of magnitude greater than TCE by the ingestion route. Biotransformation is dependent upon a variety of factors such as availability of organic chemicals, oxidation/reduction conditions, availability of microorganisms, growth factors, toxicity, and inhibition. Consequently the extent to which biotransformation may occur in groundwater at a particular site will vary and the degree to which it 
Figure 4

\begin{tabular}{|c|c|c|c|}
\hline \multirow{2}{*}{$\begin{array}{l}\text { Time of Exposure } \\
\text { Initiation (Years) }\end{array}$} & \multirow{2}{*}{$\begin{array}{c}\text { [TCE] at Start } \\
\text { of Exposure } \\
(\mathrm{mg} / \mathrm{l}) \\
\end{array}$} & \multicolumn{2}{|c|}{ Lifetime Upper Bound Cancer Risk } \\
\hline & & Scenario 1 & Scenario 2 \\
\hline$t_{o}$ & .36 & $1.1 \times 10^{-4}$ & $3.5 \times 10^{-6}$ \\
\hline 1 & .23 & $7.1 \times 10^{-5}$ & $2.2 \times 10^{-6}$ \\
\hline 2 & .14 & $4.5 \times 10^{-5}$ & $1.4 \times 10^{-6}$ \\
\hline 3 & .09 & $2.8 \times 10^{-5}$ & $8.8 \times 10^{-7}$ \\
\hline 4 & .06 & $1.8 \times 10^{-5}$ & \\
\hline 5 & .04 & $1.1 \times 10^{-5}$ & \\
\hline 6 & .02 & $7.2 \times 10^{-6}$ & \\
\hline 7 & .01 & $4.6 \times 10^{-6}$ & \\
\hline 8 & .009 & $2.9 \times 10^{-6}$ & \\
\hline 9 & .006 & $1.8 \times 10^{-6}$ & \\
\hline 10 & .004 & $1.1 \times 10^{-6}$ & \\
\hline
\end{tabular}

occurs is difficult to quantify. Use of a risk assessment for TCE without considering the potential impact of vinyl chloride could result in an underestimation of risks at the site. This could lead to risk management decisions from an inaccurate data base which could be ultimately expressed as lingering liability even after cleanup had been accomplished.

\section{Summary}

In summary it has been shown that risk assessments are generally conservative evaluations primarily due to selection of assumptions to compensate for data limitations and uncertainties. The methods selected to estimate exposure and to quantify exposure point concentrations may have a substantial effect upon the estimation of risk associated with exposure to contaminants in groundwater. Most often more accurate data will provide risk assessment outcomes that are less conservative; though use of the shower model and chemical transformation to more potent chemicals can have the opposite impact. There is no question that the best hazardous waste site clean up decisions must rely on the most accurate risk assessments possible; thereby stressing the importance of accurate initial site characterization. The implications of overestimating potential risks associated with contaminants present in groundwater or other media may result in implementation of expensive remediation that could have otherwise been less restrictive. Conversely, inaccuracies in predicting risk may also result in underestimations of exposure which may have far reaching ramifications in the areas of public health protection and liability evaluation. Currently fare more attention is focussed on costly remediation than on reducing the theoretical risk through better risk assessments. Substantial experience demonstrates that improved risk characterization is possible for most sites. This additional scientific effort is important to the process of distinguishing which sites require, the greatest attention for remediation. 


\section{Acknowledgements}

I want to thank Luci Henry, my Administrative Assistant, for assistance in preparing the manuscript and Judy L. Fauls for typing of the manuscript. In addition, I would like to thank my colleagues Gail Charnley, Sarah Foster, and Todd W. Thorslund for their constructive comments and assistance.

\section{REFERENCES}

ALBERT, R. E., TRAIN, R. E., and ANDERSON, E. L. 1977. Rationale Developed by the Environmental Protection Agency for the Assessment of Carcinogenic Risk. J. Natl. Cancer Inst. $58: 1537$.

ANDERSON, E. L., and The Carcinogen Assessment Group (CAG) of the U.S. Environmental Protection Agency. 1983. Quantitative Approaches in Use to Assess Cancer Risk. J. Risk Anal. Vol.3, No.4, p. 277.

BOUWER, E.J., RITTMANN, B. E., and McCARTY, P. L. 1981. Anaerobic degradation of halogenated 1 and 2-carbon organic compounds. Environ. Sci. Technol. 15:596-599

CLINE, P. V., and VISTE, D. R. 1984. Migration and Degradation Patterns of Volatile Organic Compounds. National Conference of Uncontrolled Hazardous Waste Sites Proceedings. P. 217.

CRUMP, K.S., GUESS, H. A., and DEAL, L. L. 1977. Confidence Intervals and Test of Hypotheses Concerning Dose-response Relations Inferred from Animal Carcinogenicity Data. Biometrics 33:437.

CRUMP, K.S., and WATSON, W. W. 1979. A Fortran Program to Extrapolate Dichotomous Animal Carcinogenicity Data to Low Doses, National Institute of Environmental Health Sciences, Contract No.1-ES-2123.

CRUMP, K. S. 1981. An Improved Procedure for Low-Dose Carcinogenic Risk Assessment from Animal Data. J. Environ. Path. Toxicol. 52:675.

DOMENICO, P. A., and PALCIAUSKAS, V. V. 1982. Alternative Boundaries in Solid Waste Management. Groundwater 20:303.

ENFIELD, C. J., CARSEL, R. F., COHEN, S. Z., PHAN, T., and WALTERS, D. M. 1982. Approximating Pollutant Transport to Groundwater. Groundwater. 20(6):711-722.

Environmental Protection Agency (EPA). 1976. Interim Procedures and Guidelines for Health Risks and Economic Impact Assessments of Suspected Carcinogens. Fed. Reg. 41:21402 (25 May 1976).

Environmental Protection Agency/Carcinogen Assessment Group (EPA/CAG). 1977. Preliminary Report on Ethylene Bisdithiocarbamate (EBDC).

Environmental Protection Agency (EPA). 1984. Users Manual for the Pesticide Root Zone Model (PRZM), Release 1. Prepared by Carsel, R. F. et al. for Technology Development and Application Branch, Environmental Research Laboratory, Athens, Georgia. December 1984. EPA-600/384-109.

Environmental Protection Agency (EPA). 1985. Final Exclusions and Final Vertical and Horizontal Spread Model (VHS). Fed. Reg. 50:229.

Environmental Protection Agency (EPA). 1985a. Endangerment Assessment Handbook. Prepared by PRC, Environmental Management, Inc., for Office of Waste Programs Enforcement, Washington, D.C. August 1985.

Environmental Protection Agency (EPA). 1986a. Guidelines for Carcinogen Risk Assessment. Fed. Reg. 51(185):33991. (24 September 1986). 
Environmental Protection Agency/Office of Pesticides and Toxic Substances (EPA/OPTS). 1986b. Report of the Dioxin Update Committee.

Environmental Protection Agency (EPA). 1986c. Guidelines for the health risk assessment of chemical mixtures. Federal Register 51:34014.

Environmental Protection Agency (EPA). 1986d. Superfund Public Health Evaluation Manual. Prepared by ICF, Inc., for Office of Emergency and Remedial Response, Washington, D.C. October 1986. EPA 540/186/060.

Environmental Protection Agency (EPA). 1986e. Guidelines for Groundwater Classification under the EPA Groundwater Protection Strategy. Office of Groundwater Protection, Office of Water. Washington, D.C.

Environmental Protection Agency (EPA). 1987f. Federal Register 50(38):7896-7900

FOSTER, S., and CHROSTOWSKI, P. 1987. Inhalation exposures to volatile organic contaminants in the shower. Presented at 80th annual meeting of the Air Pollution Control Association, New York, June 21.

FREEZE, R. A., and CHERRY, J. A. 1979. Groundwater. Prentice-Hall, Inc. Englewood Cliffs, New Jersey.

Interagency Regulatory Liaison Group (IRLG). 1979. Scientific Basis for the Identification of Potential Carcinogens and Estimation of Risks. J. Natl. Cancer Inst. 63:243.

International Agency for Research on Cancer (IARC). 1982. Monographs on the evaluation of the carcinogenic risk of chemicals to humans. Supplement 4, Chemicals and Industrial Processes Associated with Cancer in Humans. Lyon, France. P. 7.

KOBAYASHI, H., and RITTMANN, B. E. 1982. Microbial Remediation of Hazardous Organic Compounds. Environ. Sci. Technol. 16:170A-183A.

LEVINE, T., RISPIN, A., SCOTT, C. S., MARCUS, W., CHEN, C., and LIBB, H. 1987. Special Report on Ingested Inorganic Arsenic: Skin Cancer; Nutritional Essentiality. Draft for U.S. Environmental Protection Agency Science Advisory Board Review.

MOOLGAVKAR, S. H., and KNUDSON, A. G. 1981. Mutation and cancer: A model for human carcinogenesis. J. Natl. Cancer Inst. 66:1037.

MOORE, J. A. 1987. Recommended Agency Policy on the Carcinogenicity Risk Associated with the Ingestion of Inorganic Arsenic - Action Memorandum. U.S. Environmental Protection Agency. Office of Pesticides and Toxic Substances.

National Academy of Sciences/National Research Council (NAS/NRC). 1983. Risk Assessment in the Federal Government: Managing the Process. Prepared by the Committee on the Institutional Means for Assessment of Risk to Public Health, Commission on Life Sciences (National Academy Press, Washington, D. C.).

Office of Science and Technology Policy (OSTP). 1985. Chemical Carcinogens: Review of the Science and Its Associated Principles. Fed. Reg. 50:10372.

PARSONS, F., WOOD, P. R., DeMARCO, J. 1984. Transformation of Tetrachloroethene and Trichloroethane in Microcosms and Groundwater. Res. Technol. P. 56.

PATRICK, D., and PETERS, W.D. 1985. Exposure assessment in setting air pollution regulations: ASARCO, Tacoma, a case study. Presented at the Society for Risk Analysis annual meeting. Washington, D.C.

SAWHNEY, B. L., PIGNATELlo,J. J., and STEINBERG, S. M. 1988. Determination of 1,2Dibromoethane (EDB) in Field Soils: Implications for Volatile Organic Compounds. J. Environ. Qual. Vol. 17, No., 1, p. 149. 
SEHMEL, G. A., and HODGSON, W. H. 1979. A Model for Predicting Dry Deposition of Particles and Gases to Environmental Surfaces. Prepared for the U.S. Department of Energy by Pacific Northwest Laboratory. October. PNL-SA-6271-REV 1.

SHABECOFF, P. 1988. EPA reassesses the cancer risk of many chemicals. New York Times. Monday, January 4. P. A1.

THORSLUND, T. W., CHARNLEY, G. and ANDERSON, E. L. 1986. Innovative Use of Toxicological Data to Improve Cost-Effectiveness of Waste Cleanup. Presented at the Superfund '86: Management of Uncontrolled Hazardous Waste Sites, Washington, D.C. December 1-3.

THORSLUND, T. W., BROWN, C. C., and CHARNLEY, G. 1987. Biologically motivated cancer risk models. Risk Anal. 7:109.

UMBREIT, T. H., HESSE, E. G., and GALLO, M. A. 1986. Bioavailability of dioxin in soil from a 2,4,5-T manufacturing site. Science 232:497.

VAN DEN BERG, M., van GREEVENBROEK, M., OLIE, K., and HUTZINGER, O. 1986. Bioavailability of PCDDs and PCDFs on fly ash after semi-chronic ingestion by rat. Chemosphere 15:509.

VOGEL, T. M., and McCARTY, P. L. 1985. Biotransformation of tetrachloroethylene to trichloroethylene, dichloroethylene, vinyl chloride, and carbon dioxide under methanogenic conditions. Appl. Environ. Microbiol. 49:1080-1083.

WALTON, W. C. 1985. Practical Aspects of Groundwater Modeling. Second Edition. National Water Well Association, Worthington, Ohio. 